\title{
Fermionic quasinormal modes for two-dimensional Hořava-Lifshitz black holes
}

\author{
M. M. Stetsko ${ }^{\mathrm{a}}$ \\ Department for Theoretical Physics, Ivan Franko National University of Lviv, 12 Drahomanov Str., Lviv 79005, Ukraine
}

Received: 29 December 2016 / Accepted: 11 June 2017 / Published online: 20 June 2017

(C) The Author(s) 2017. This article is an open access publication

\begin{abstract}
To obtain fermionic quasinormal modes, the Dirac equation for two types of black holes is investigated. It is shown that two different geometries lead to distinctive types of quasinormal modes, while the boundary conditions imposed on the solutions in both cases are identical. For the first type of black hole, the quasinormal modes have continuous spectrum with negative imaginary part that provides the stability of perturbations. For the second type of the black hole, the quasinormal modes have a discrete spectrum and are completely imaginary.
\end{abstract}

\section{Introduction}

The investigation of gravitational perturbations of the Schwarzschild geometry started several decades ago [1-3]. That idea was applied for the examination of perturbations of other types of black holes caused by the fields of a different nature, for example scalar field or Dirac field. All this work gave birth to the method which is known nowadays as the quasinormal mode method (QNM) [4-6]. This method allows one to get important information as regards the stability of black holes against perturbations of different types which evolve in the exterior region of the black holes. We also note that in most cases the influence of the external fields is considered perturbatively and the backreaction of the field on the black hole's metric is not taken into account. The quasinormal modes and their quasinormal frequencies are useful for different branches of investigations in general relativity. In particular, QN modes are important in gaugestring duality theories (AdS-CFT) [7] because they define the relaxation times of dual field theories [8]. The relation between QN modes and retarded correlators of dual field theories was also established [9-12]. Another possibility is

\footnotetext{
a e-mail: mstetsko@gmail.com
}

due to Hod's conjecture as regards the quantization of a black hole's area [13-15]. The connection between QN modes and Hawking radiation is also considered [16,17]. The progress in experimental astrophysics and the discovery of gravitational waves opened a new perspective for application of the QNM method for the estimation of different parameters of compact sources of gravitational field or the verification of some conjectures of general relativity [5].

Another area of active research is related to two different disciplines, namely quantum mechanics and general relativity. A well-known and still open problem is the reconciliation of the principles of these theories. It might give some hints about the underlying theory of quantum gravity. For example, nonrenormalizability is a crucial problem when one tries to quantize general relativity in the way possible for other gauge fields. To overcome this difficulty, it was supposed that the general relativity should be treated as an effective theory and, in order to have a gravitation theory suitable for quantization, the principles of general relativity should be elaborated. One of the approaches that leads to power-countable UV-renormalizability is the so-called Hořava-Lifshitz (HL) theory [18-20]. General relativity can be recovered as an infrared limit of the Hořava-Lifshitz theory. Because of its attractive and promising features, the Hořava-Lifshitz approach has gained considerable interest in recent years. In particular, black hole solutions were found and their properties were investigated [21-29]. The quasinormal modes for HL black holes were studied in Refs. [30-34].

The examination of the evolution of the fields in a background of lower-dimensional black holes is an interesting and important problem. Firstly, because of the simplicity of those problems in comparison with higher-dimensional cases, analytical computations can be made and, as a result, for many kinds of black holes exact $\mathrm{QN}$ frequencies can be calculated. The second important point is the fact that lowerdimensional black holes and the fields evolving in their backgrounds give some hints or reveal some aspects of higher- 
dimensional cases. The other important moment which stimulates the interest to lower-dimensional black holes is the fact that they might be suitable models for analogue gravity [35].

Our paper is organized as follows: in Sect. 2 we briefly review some $1+1$-dimensional black hole solutions in HL gravity. In Sect. 3 the Dirac equations for fermion fields in specific black hole backgrounds are written. In the Sect. 4 we investigate fermionic QNMs for chosen BH metrics. The last section contains some concluding remarks.

\section{1 + 1-dimensional black holes solutions in Hořava theory}

$1+1$-dimensional black holes in Hořava gravity were considered in Ref. [37]. The starting point is two-dimensional action integral which in the case of Hořava-Lifshitz (HL) gravity takes the form

$S=\frac{M_{P l}^{2}}{2} \int \mathrm{d} t \mathrm{~d} x\left(-\frac{1}{2} \eta N^{2} a^{2}+\alpha N^{2} \varphi^{\prime 2}-V(\varphi)\right)$

and here $\alpha, \eta$ are constants and $a=N^{\prime} / N=(\ln N)^{\prime}$.

The black hole solution in two-dimensional HL gravity is described by a shift function which can be represented as follows:

$N^{2}(x)=2 C_{2}+\frac{A}{\eta} x^{2}-2 C_{1} x+\frac{B}{\eta x}+\frac{C}{3 \eta x^{2}}$

where $A, B, C, C_{1}, C_{2}$ are some constants. The scalar field can be written in the form

$\varphi(x)=\frac{1}{2} \ln \left(2 C_{2}+\frac{A}{\eta} x^{2}-2 C_{1} x+\frac{B}{\eta x}+\frac{C}{3 \eta x^{2}}\right)$.

The expressions for the shift function as well as the scalar potential are quite general and we consider some particular cases, taking specific values for the constants mentioned above. We describe them in the following sequence.

- The first case: for $C_{1}=-M, C_{2}=-\frac{1}{2}$ and $A=B=$ $C=0$ we have $V_{\varphi}(\varphi)=0$ (or $V(\varphi)=$ const $)$ and one arrives at the solution:

$\mathrm{d} s^{2}=-(2 M|x|-1) \mathrm{d} t^{2}+\frac{1}{(2 M|x|-1)} \mathrm{d} x^{2}$.

It should be noted that similar solution was obtained in the context of the ordinary $1+1$-dimensional gravity [38].

- The second case: the constants are chosen in the following way: $A=\Lambda, B=C=0, C_{1}=-M$ and $C_{2}=-\epsilon / 2$. For this case we have $V_{\varphi}(\varphi)=\Lambda$, which leads to linear dependence for the scalar potential $V(\varphi)=\Lambda \varphi$. The solution takes the form

$$
\begin{aligned}
\mathrm{d} s^{2}= & -\left((\Lambda / \eta)^{2} x^{2}+2 M x-\epsilon\right) \mathrm{d} t^{2} \\
& +\frac{1}{\left((\Lambda / \eta)^{2} x^{2}+2 M x-\epsilon\right)} \mathrm{d} x^{2} .
\end{aligned}
$$

The latter metric can be rewritten in a bit different form after some kind of transformation of coordinates [34]:

$u=\sqrt{\frac{\Lambda}{\eta}} x+\sqrt{\frac{\eta}{\Lambda}} M$.

Having used the above transformation we arrive at a new representation of the metric (5):

$\mathrm{d} s^{2}=-\left(u^{2}-u_{+}^{2}\right) \mathrm{d} t^{2}+\frac{l^{2}}{\left(u^{2}-u_{+}^{2}\right)} \mathrm{d} u^{2}$

and here $u_{+}=\sqrt{(\eta / \Lambda) M^{2}+\epsilon}$ and $l=\sqrt[4]{\Lambda / \eta}$. It is worth of note that in the new coordinate system the horizon of the black hole is located at the point $u=u_{+}$.

- The third case: the so-called Schwarzschild-like solution. In this case one imposes the requirement that $A=C=$ $C_{1}=0, B=-2 M, C_{2}=1 / 2$ and $\eta=1$. As a result the metric would look as follows:

$N^{2}(x)=1-\frac{2 M}{x}, \quad \varphi(x)=\frac{1}{2} \ln \left(1-\frac{2 M}{x}\right)$.

We also note that in this case the potential can be written in explicit form [37]. So the metric takes the Schwarzschild-like form

$\mathrm{d} s^{2}=-\left(1-\frac{2 M}{x}\right) \mathrm{d} t^{2}+\frac{1}{\left(1-\frac{2 M}{x}\right)} \mathrm{d} x^{2}$.

- The fourth case is the so-called Reissner-Nordström-like case. The constants should be chosen as follows: $A=$ $C_{1}=0, B=-2 M, C=3 Q^{2}$ and $C_{2}=1 / 2$. So we obtain

$N^{2}(x)=1-\frac{2 M}{x}+\frac{Q^{2}}{x^{4}}, \quad \varphi(x)=\frac{1}{2} \ln \left(1-\frac{2 M}{x}+\frac{Q^{2}}{x^{4}}\right)$.

As a result the metric takes the Reissner-Nordström-like form

$\mathrm{d} s^{2}=-\left(1-\frac{2 M}{x}+\frac{Q^{2}}{x^{2}}\right) \mathrm{d} t^{2}+\frac{1}{\left(1-\frac{2 M}{x}+\frac{Q^{2}}{x^{2}}\right)} \mathrm{d} x^{2}$. 
We note that in contrast to the previous cases here, it is not possible to find explicit form for the scalar potential.

\section{Dirac equation}

Fermionic perturbations in the background of two-dimensional black holes is governed by Dirac equation. Supposing that the fermionic fields are chargeless, we can write

$$
\left(\gamma^{\mu} \nabla_{\mu}+m\right) \psi=0
$$

where $m$ is the mass of fermionic field $\psi$. The covariant derivative is defined as follows:

$\nabla_{\mu}=\partial_{\mu}+\frac{1}{2} \omega_{\mu}^{A B} J_{A B}$

where $J_{A B}=\frac{1}{4}\left[\gamma_{A}, \gamma_{B}\right]$ are the Lorentz group generators and $\omega_{\mu}^{A B}$ denotes components of spin connection. Gamma matrices in a curved space take the form $\gamma^{\mu}=e_{A}^{\mu} \gamma^{A}$ where $e_{A}^{\mu}$ are the diad components and $\gamma^{A}$ are the gamma matrices for flat space-time. To obtain the spin connection, the Cartan structure equations should be utilized,

$d e^{A}+\omega^{A}{ }_{B} \wedge e^{B}=0$.

The connection is supposed to be torsionless. It can easily be verified that for all the cases we have mentioned above the only nonzero component of the spin connection is the component $\omega^{01}$. Here we will consider the first two cases and the others will be investigated elsewhere.

\subsection{Dirac equation for the first kind of the metric}

In this case the diad takes the form

$e^{0}=\sqrt{2 M x-1} \mathrm{~d} t, \quad e^{1}=\frac{1}{\sqrt{2 M x-1}} \mathrm{~d} x$.

Having used Eq. (14), we obtain the spin connection:

$\omega^{0}{ }_{1}=\frac{M}{\sqrt{2 M x-1}} e^{0}$.

For gamma matrices $\gamma^{A}$ (Lorentzian) the following representation will be used:

$\gamma^{0}=i \sigma^{2}, \quad \gamma^{1}=\sigma^{1}$

where $\sigma^{i}$ are the Pauli matrices. In the curvilinear coordinates, the gamma matrices look as follows:

$\gamma^{t}=\frac{1}{\sqrt{2 M x-1}} \gamma^{0}, \quad \gamma^{x}=\sqrt{2 M x-1} \gamma^{1}$.

Now the Dirac equation for the metric (4) can be written:

$$
\left(\frac{i \sigma^{2}}{\sqrt{2 M x-1}}\left(\partial_{t}-\frac{M}{2} \sigma^{3}\right)+\sigma^{1} \sqrt{2 M x-1} \partial_{x}+m\right) \psi=0 .
$$

We suppose that the solution of the equation can be represented in the form

$$
\psi(t, x)=\frac{1}{\sqrt[4]{2 M x-1}} e^{-i \omega t}\left(\begin{array}{l}
\psi_{1} \\
\psi_{2}
\end{array}\right) .
$$

The system of equations for the components of the spinor part of the wavefunction takes the form

$$
\begin{aligned}
& \frac{-i \omega}{\sqrt{2 M x-1}} \psi_{2}+\sqrt{2 M x-1} \partial_{x} \psi_{2}+m \psi_{1}=0, \\
& \frac{i \omega}{\sqrt{2 M x-1}} \psi_{1}+\sqrt{2 M x-1} \partial_{x} \psi_{1}+m \psi_{2}=0 .
\end{aligned}
$$

The system of the equations we have obtained can be decoupled and we write the equation for one component of the wavefunction. For example for the function $\psi_{1}$ :

$$
(2 M x-1) \partial_{x}^{2} \psi_{1}+M \partial_{x} \psi_{1}+\left(\frac{\omega^{2}-i \omega M}{2 M x-1}-m^{2}\right) \psi_{1}=0 .
$$

\subsection{Dirac equation for the second kind of the metric}

Now we consider the Dirac equation for the second kind of the metric; see (5). The transformed form of the metric represented by Eq. (7) will be used here. The diad field for this metric takes the form

$e^{0}=\sqrt{u^{2}-u_{+}^{2}} \mathrm{~d} t, \quad e^{1}=\frac{l \mathrm{~d} u}{\sqrt{u^{2}-u_{+}^{2}}}$.

Having used the Cartan structure equations (14), we obtain the following expression for the spin connection form:

$\omega^{0}{ }_{1}=\frac{u}{l \sqrt{u^{2}-u_{+}^{2}}} e^{0}$.

Finally, the Dirac equation for the background metric (7) can be written as follows:

$$
\left(\frac{l}{\sqrt{u^{2}-u_{+}^{2}}} i \sigma^{2}\left(\partial_{t}-\frac{u}{2 l} \sigma^{3}\right)+\sqrt{u^{2}-u_{+}^{2}} \sigma^{1} \partial_{u}\right) \psi+\bar{m} \psi=0
$$

where $\bar{m}=l m$. Similarly to the previous case, in order to simplify the procedure of the solution of the above Dirac equation, we assume that the spinor wavefunction takes the form

$\psi=\frac{e^{-i \omega t}}{\sqrt[4]{u^{2}-u_{+}^{2}}}\left(\begin{array}{l}\psi_{1} \\ \psi_{2}\end{array}\right)$.

Having substituted the wavefunction (26) into Eq. (25), and after a little algebra, we obtain the system of equations for the components of spinor wavefunction $\psi_{1}$ and $\psi_{2}$ : 


$$
\begin{gathered}
\left(-\frac{i l \omega}{\sqrt{u^{2}-u_{+}^{2}}}+\sqrt{u^{2}-u_{+}^{2}} \partial_{u}\right) \psi_{2}+\bar{m} \psi_{1}=0, \\
\left(\frac{i l \omega}{\sqrt{u^{2}-u_{+}^{2}}}+\sqrt{u^{2}-u_{+}^{2}} \partial_{u}\right) \psi_{1}+\bar{m} \psi_{2}=0 .
\end{gathered}
$$

The system of equations can easily be decoupled and, as a result, the equation for any component of the spinor wavefunction (26) can be obtained. We write the equation for the upper component $\psi_{1}$ :

$$
\left(\left(u^{2}-u_{+}^{2}\right) \partial_{u}^{2}+u \partial_{u}+\frac{\bar{\omega}^{2}-i \bar{\omega} u}{u^{2}-u_{+}^{2}}-\bar{m}^{2}\right) \psi_{1}=0
$$

and here $\bar{\omega}=l \omega$.

\section{Quasinormal modes}

In this section, the Dirac equations for two cases of the metric will be examined again separately. We will find the quasinormal modes and then compare the results. It should be remarked that the quasinormal modes for scalar perturbations in the same black hole background were considered in Ref. [34].

\subsection{Quasinormal modes for the metric of the first kind}

In this subsection, we will consider Eq. (22) and investigate the quasinormal modes for it. Firstly, we perform a transformation of coordinates and introduce a new one instead of coordinate $x$ by the following relation:

$z=\frac{m}{M} \sqrt{2 M x-1}$.

Equation (22) can be rewritten in the form

$\partial_{z}^{2} \psi_{1}+\frac{\tilde{\omega}^{2}-i \tilde{\omega}}{z^{2}} \psi_{1}-\psi_{1}=0$

and here $\tilde{\omega}=\omega / M$. We suppose that the wavefunction $\psi_{1}$ of the latter equation takes the form

$\psi_{1}=\sqrt{z} F(z)$.

Having performed that transformation, we arrive at the modified Bessel equation:

$z^{2} F^{\prime \prime}+z F^{\prime}-\left(v^{2}+z^{2}\right) F=0$

where $v=1 / 2+i \tilde{\omega}$. The solutions of the latter equation are the well-known modified Bessel functions [42] and can be used for analysis of quasinormal modes:

$F(z)=A I_{v}(z)+B K_{v}(z)$.
To obtain quasinormal modes, boundary conditions on the solutions of the wave equation have to be imposed. It is well known that in the vicinity of the horizon the solution of the corresponding wave equation should behave as an ingoing wave. The behaviour of the wavefunction at infinity depends on the background metric. In case of an asymptotically flat geometry the solution of the wave equation should behave as an outgoing wave. In our case the geometry is not asympotically flat; the metric function diverges at infinity. We impose the requirement that the wavefunction of our equation (33) tend to zero at infinity. The function $I_{v}(z)$ is divergent when $z \rightarrow+\infty$ for arbitrary value of the parameter $v$, whereas $K_{v}(z)$ has nondivergent behaviour. To remove the term divergent at infinity we put $A=0$. As a result, the solution which fulfills the boundary condition at infinity takes the form

$F(z)=B K_{v}(z)$

In the vicinity of the horizon we use asymptotic formula for the Bessel function $K_{v}(z)$ [42]:

$K_{v}(z) \simeq \frac{1}{2} \Gamma(v)\left(\frac{z}{2}\right)^{-v}$.

The above decomposition is valid when $\operatorname{Re}(v)>0$. The latter condition leads to the restriction on the imaginary part of the frequency, namely $\operatorname{Im}(\omega)=\omega_{I}<M / 2$. For the upper component of the Dirac wavefunction we obtain

$\psi_{u p} \sim e^{-i \omega t} z^{-i \tilde{\omega}}=e^{-i \omega(t+1 / M \ln z)}$.

As one can see in the domain close to the horizon the upper component of the Dirac wavefunction behaves as an ingoing wave as it should be for the quasinormal modes. To make them stable one should impose the requirement that the imaginary part of the quasinormal modes be negative. When $\operatorname{Re}(v)<0$ we use the well-known relation for the Bessel function $K_{v}(z)$ :

$K_{v}(z)=K_{-v}(z)$

And in the domain close to the horizon we also use the approximate relation

$K_{-v}(z) \simeq \frac{1}{2} \Gamma(-v)\left(\frac{z}{2}\right)^{v}$.

It was shown that in this case near the horizon the upper component for the Dirac wavefunction behaves as an outgoing wave so it does not satisfy the boundary condition for the quasinormal modes [39]. To analyze the behaviour of the lower component we use Eq. (21) and write

$$
\begin{aligned}
\psi_{2} & =-\left(\frac{i \tilde{\omega}}{z}+\frac{\partial}{\partial z}\right) \psi_{1}=-z^{-i \tilde{\omega}} \frac{\partial}{\partial z} z^{i \tilde{\omega}} \psi_{1} \\
& =-z^{1 / 2-v} \frac{\partial}{\partial z} z^{v} F(z) .
\end{aligned}
$$


Having substituted the solution (35) into the above relation and taking into account the relations for the derivatives of the Bessel function we obtain

$\psi_{2}=-B z^{1 / 2} K_{v-1}(z)$.

The lower component (41) of the Dirac wavefunction similarly to the upper one tends to zero at infinity. To examine the behaviour in the domain close to the horizon we again use Eq. (36) and write

$\psi_{2} \simeq-\frac{B}{2} \Gamma(v-1) z^{1 / 2}\left(\frac{z}{2}\right)^{-v+1}$.

The relation holds when $\operatorname{Re}(v-1)>0$, which is equivalent to the condition $\omega_{I}<-\frac{M}{2}$. Similarly to the upper component, the lower component also behaves as an ingoing wave in the vicinity of the horizon:

$\psi_{\text {down }} \simeq z e^{-i \omega(t+1 / M \ln z)}$.

When $\operatorname{Re}(v-1)<0$ Eq. (38) can be used again, but it can be shown that in this case one obtains outgoing waves close to the horizon which does not satisfy boundary condition at the horizon. We can conclude that both components of the Dirac wavefunction might satisfy the necessary conditions for the quasinormal modes and to make them stable we have to impose $\omega_{I}<-M / 2$. We note that a similar conclusion was made in Ref. [39]. It is generally supposed that quasinormal modes should have discrete spectrum, but this feature of the spectrum does not follow with necessity from the imposed boundary conditions on the quasinormal modes. A continuous spectrum for gravitational perturbations of scalar type was obtained for the RN-AdS black hole in the five-dimensional case [40]. A continuous spectrum for the quasinormal frequencies was also obtained for the twodimensional acoustic black hole in the case of analogue gravity [41]. We also remark that for integer $v$ one of the solutions of Eq. (33) namely the function $K_{n}(z)$ can be introduced as limit of the function $K_{v}(z)$ when $v \rightarrow n$. The behaviour of the solution $K_{n}(z)$ at infinity and at the horizon is similar to the case of noninteger $v$ and it means that it also satisfies the above mentioned conditions for the quasinormal modes, but there is no specific requirement that might distinguish integer values of the parameter $v$ from noninteger ones.

In the case of scalar particles the behaviour of the particle flux at infinity was also analyzed [34]. Taking into account the definition of the flux for the Dirac particles we can write

$\mathcal{F}=\sqrt{-g} \bar{\psi} \gamma^{r} \psi$

and here $\gamma^{r}=e_{1}^{r} \gamma^{1}, \bar{\psi}=\psi^{\dagger} \gamma^{0}, \sqrt{-g}=1$. As a consequence we obtain

$\mathcal{F}=\left|\psi_{1}\right|^{2}-\left|\psi_{2}\right|^{2}$.

The behaviour of the Dirac flux at infinity is completely defined by the corresponding behaviour of the upper and lower components of the Dirac wavefunction. Because both of them tend to zero at infinity the Dirac flux vanishes at infinity. It is worth being emphasized that in case of scalar particles the vanishing behaviour of the flux might be provided when one imposes that the scalar field vanishes at infinity (Dirichlet condition) or its derivative disappears at infinity (Neumann condition). For Dirac particles there is no specific requirement for the derivatives of upper or lower components of the Dirac wavefunction but both of them are connected through the relations (21) or (40) where derivatives from components are present, so in some way boundary conditions on the upper and lower components are equivalent to imposing both Neumann and Dirichlet conditions.

\subsection{Quasinormal modes for the second kind of the metric}

Similarly to the previous case, we have to solve the equation (29). The equation can be rewritten in the form of a standard hypergeometric equation. To simplify the calculations, we perform a transformation of coordinates defined by the following relation:

$z=\frac{u-u_{+}}{u+u_{+}}$

It can easily be verified that the domain of variation of the variable $z$ is the interval: $-1 \leqslant z \leqslant 1$ and since we consider the motion of the particle outside the black hole our domain will be as follows: $0 \leqslant z \leqslant 1$. Having used the transformation (46) we rewrite the equation (29) in the form

$$
\begin{aligned}
& z(1-z)^{2} \psi_{1}^{\prime \prime}+(1-z)\left(\frac{1}{2}-\frac{3}{2} z\right) \psi_{1}^{\prime} \\
& +\left(\frac{\bar{\omega}^{2}(1-z)^{2}}{z}-\frac{i \bar{\omega}}{2 z}(1-z)(1+z)-\bar{m}^{2}\right) \psi_{1}=0 .
\end{aligned}
$$

We suppose that the wavefunction $\psi_{1}$ can be represented in the form

$\psi_{1}=z^{\alpha}(1-z)^{\beta} F(z)$.

As a consequence, a hypergeometric equation for the function $F(z)$ can be written

$z(1-z) F^{\prime \prime}(z)+(c-(a+b+1) z) F^{\prime}(z)-a b F(z)=0$

where the coefficients $a, b$ and $c$ are given by the relations

$$
\begin{aligned}
& a+b=2(\alpha+\beta)+\frac{1}{2}, \\
& a b=(\alpha+\beta)^{2}+\frac{1}{2}(\alpha+\beta)+\bar{\omega}^{2}+\frac{i}{2} \bar{\omega}, \\
& c=2 \alpha+\frac{1}{2} .
\end{aligned}
$$


The parameters should satisfy the system of equations:

$$
\begin{aligned}
& \alpha^{2}-\frac{\alpha}{2}+\bar{\omega}^{2}-\frac{i}{2} \bar{\omega}=0, \\
& \beta^{2}-\alpha^{2}+\frac{\alpha}{2}-\bar{\omega}^{2}+\frac{i}{2} \bar{\omega}-\bar{m}^{2}=0 .
\end{aligned}
$$

The latter system of equations can be solved easily and we obtain

$$
\begin{aligned}
& \alpha_{1}=i \bar{\omega}+\frac{1}{2}, \quad \alpha_{2}=-i \bar{\omega}, \\
& \beta_{1}=\bar{m}, \quad \beta_{2}=-\bar{m} .
\end{aligned}
$$

We note that any combination of $\alpha$ and $\beta$ can be chosen and substituted into the system of equations (50). We consider different combinations of the parameters and analyze the solution we arrive at. Let us start from the combination of parameters $\alpha_{1}=i \bar{\omega}+\frac{1}{2}$ and $\beta_{1}=\bar{m}$. As a result from the system (50) we obtain

$a=\bar{m}+\frac{1}{2}+2 i \bar{\omega}, \quad b=\bar{m}+1, \quad c=2 i \bar{\omega}+\frac{3}{2}$.

It is well known that the general solution of the hypergeometric equation (49) can be represented in the form [42]

$$
\begin{aligned}
F= & A_{2} F_{1}(a, b, c ; z)+B z^{1-c} F_{1} \\
& \times(a-c+1, b-c+1,2-c ; z) .
\end{aligned}
$$

The combination of parameters $a, b, c(57)$ and the above general solution of the hypergeometric equation (58) immediately leads to the solution of the hypergeometric equation (49), which takes the form

$$
\begin{aligned}
F= & A_{2} F_{1}\left(\bar{m}+\frac{1}{2}+2 i \bar{\omega}, \bar{m}+1,2 i \bar{\omega}+\frac{3}{2} ; z\right) \\
& +B z^{-1 / 2-2 i \bar{\omega}}{ }_{2} F_{1}\left(\bar{m}, \bar{m}+\frac{1}{2}-2 i \bar{\omega}, \frac{1}{2}-2 i \bar{\omega} ; z\right) .
\end{aligned}
$$

Now we choose another combination of the parameters: $\alpha_{1}=$ $i \bar{\omega}+1 / 2$ and $\beta_{2}=-\bar{m}$. As a consequence we obtain

$a=\frac{1}{2}-\bar{m}+2 i \bar{\omega}, \quad b=1-\bar{m}, \quad c=2 i \bar{\omega}+\frac{3}{2}$.

The general solution for that combination of parameters takes the form

$$
\begin{aligned}
F= & A_{2} F_{1}\left(\frac{1}{2}-\bar{m}+2 i \bar{\omega}, 1-\bar{m}, 2 i \bar{\omega}+\frac{3}{2} ; z\right) \\
& +B z^{-1 / 2-2 i \bar{\omega}}{ }_{2} F_{1}\left(-\bar{m}, \frac{1}{2}-\bar{m}-2 i \bar{\omega}, \frac{1}{2}-2 i \bar{\omega} ; z\right) .
\end{aligned}
$$

The third variant for the parameters $\alpha$ and $\beta$ can be taken as follows: $\alpha_{2}=-i \bar{\omega}$ and $\beta_{1}=\bar{m}$. For the chosen combination we obtain

$a=\bar{m}, \quad b=\bar{m}+\frac{1}{2}-2 i \bar{\omega}, \quad c=\frac{1}{2}-2 i \bar{\omega}$.
So, we write the general solution of the hypergeometric equation in the form

$$
\begin{aligned}
F= & A_{2} F_{1}\left(\bar{m}, \bar{m}+\frac{1}{2}-2 i \bar{\omega}, \frac{1}{2}-2 i \bar{\omega} ; z\right) \\
& +B z^{1 / 2+2 i \bar{\omega}}{ }_{2} F_{1}\left(\bar{m}+\frac{1}{2}+2 i \bar{\omega}, \bar{m}+1, \frac{3}{2}+2 i \bar{\omega} ; z\right) .
\end{aligned}
$$

The last combination of the parameters $\alpha$ and $\beta$ that we can choose is $\alpha_{2}=-i \bar{\omega}$ and $\beta_{2}=-\bar{m}$. Taking this combination into consideration, we obtain

$a=-\bar{m}, \quad b=\frac{1}{2}-\bar{m}-2 i \bar{\omega}, \quad c=\frac{1}{2}-2 i \bar{\omega}$.

The corresponding general solution of the hypergeometric equation for the obtained above parameters $a, b$ and $c$ can be represented in the form

$$
\begin{aligned}
F= & A_{2} F_{1}\left(-\bar{m}, \frac{1}{2}-\bar{m}-2 i \bar{\omega}, \frac{1}{2}-2 i \bar{\omega} ; z\right) \\
& +B z^{1 / 2+2 i \bar{\omega}}{ }_{2} F_{1}\left(\frac{1}{2}-\bar{m}+2 i \bar{\omega}, 1-\bar{m}, \frac{3}{2}+2 i \bar{\omega} ; z\right) .
\end{aligned}
$$

Having used Eq. (48), we can come back to the upper component of the spinor wavefunction $\psi_{1}$. For the first combination of parameters $\alpha$ and $\beta$, we obtain

$$
\begin{aligned}
\psi_{1}= & (1-z)^{\bar{m}}\left(A z^{i \bar{\omega}+1 / 2}{ }_{2} F_{1}\left(\bar{m}+\frac{1}{2}+2 i \bar{\omega}, \bar{m}+1,2 i \bar{\omega}+\frac{3}{2} ; z\right)\right. \\
& \left.+B z^{-i \bar{\omega}}{ }_{2} F_{1}\left(\bar{m}, \bar{m}+\frac{1}{2}-2 i \bar{\omega}, \frac{1}{2}-2 i \bar{\omega} ; z\right)\right) .
\end{aligned}
$$

For the second combination of the parameters $\alpha$ and $\beta$, one arrives at

$$
\begin{aligned}
\psi_{1}= & (1-z)^{-\bar{m}}\left(A z^{i \bar{\omega}+1 / 2}{ }_{2} F_{1}\left(\frac{1}{2}-\bar{m}+2 i \bar{\omega}, 1-\bar{m}, 2 i \bar{\omega}+\frac{3}{2} ; z\right)\right. \\
& \left.+B z^{-i \bar{\omega}}{ }_{2} F_{1}\left(-\bar{m}, \frac{1}{2}-\bar{m}-2 i \bar{\omega}, \frac{1}{2}-2 i \bar{\omega} ; z\right)\right) .
\end{aligned}
$$

It can be shown that the upper function $\psi_{1}$ corresponding to the third variant of parameters $\alpha$ and $\beta$ is completely the same as the function (66). The same holds for the function (67) and the upper function that appears for the fourth variant of parameters $\alpha$ and $\beta$. To find a link between the functions (66) and (67), a well-known relation for the hypergeometric functions should be used [42]:

${ }_{2} F_{1}(a, b, c ; z)=(1-z)^{c-a-b}{ }_{2} F_{1}(c-a, c-b, c ; z)$.

Having applied it to the function (67), we immediately arrive at the conclusion that the upper functions (66) and (67) are completely the same. So, all the variants for the parameters $\alpha$ and $\beta$ lead to the unique upper function $\psi_{1}$, which can be taken in the form (66).

To obtain quasinormal modes the behaviour of the wavefunction (66) should be analyzed at the horizon point and 
at infinity. In the vicinity of the horizon point $(z=0)$, the wavefunction $\psi_{1}$ behaves as

$$
\psi_{1} \simeq A z^{i \bar{\omega}+1 / 2}+B z^{-i \bar{\omega}}=A e^{(i \bar{\omega}+1 / 2) \ln z}+B e^{-i \bar{\omega} \ln z} .
$$

The first term of the given above function would correspond to an outgoing wave solution, whereas the second one gives rise to the ingoing wave. According to the quasinormal mode method, it is required that only the ingoing waves exist in the neighbourhood of the horizon. It leads to the condition that $A=0$. As a result, we arrive at the expression for the upper wavefunction $\psi_{1}$ :

$$
\psi_{1}(z)=B z^{-i \bar{\omega}}(1-z)^{\bar{m}} F_{1}\left(\bar{m}+\frac{1}{2}-2 i \bar{\omega}, \bar{m}, \frac{1}{2}-2 i \bar{\omega} ; z\right) .
$$

To examine the behaviour of the wavefunction at infinity $(\mathrm{z}=1)$, a linear transformation $z \rightarrow 1-z$ should be made and Kummer's relation for the hypergeometric functions should be used [42]. Thus, we obtain

$$
\begin{aligned}
\psi_{1}(z)= & B z^{-i \bar{\omega}}(1-z)^{\bar{m}}\left(\frac{\Gamma(-2 \bar{m}) \Gamma(1 / 2-2 i \bar{\omega})}{\Gamma(-\bar{m}) \Gamma(1 / 2-\bar{m}-2 i \bar{\omega})}\right. \\
& \times{ }_{2} F_{1}\left(\bar{m}+\frac{1}{2}-2 i \bar{\omega}, \bar{m}, 2 \bar{m}+1 ; 1-z\right) \\
& +(1-z)^{-2 \bar{m}} \frac{\Gamma(2 \bar{m}) \Gamma(1 / 2-2 i \bar{\omega})}{\Gamma(\bar{m}) \Gamma(1 / 2+\bar{m}-2 i \bar{\omega})} \\
& \left.\times{ }_{2} F_{1}\left(-\bar{m}, \frac{1}{2}-\bar{m}-2 i \bar{\omega}, 1-2 \bar{m} ; 1-z\right)\right) .
\end{aligned}
$$

The asymptotic expression for the latter function in the neighbourhood of infinity takes the form

$$
\begin{aligned}
\psi_{1} \simeq & B(1-z)^{\bar{m}} \frac{\Gamma(-2 \bar{m}) \Gamma(1 / 2-2 i \bar{\omega})}{\Gamma(-\bar{m}) \Gamma(1 / 2-\bar{m}-2 i \bar{\omega})} \\
& +B(1-z)^{-\bar{m}} \frac{\Gamma(2 \bar{m}) \Gamma(1 / 2-2 i \bar{\omega})}{\Gamma(\bar{m}) \Gamma(1 / 2+\bar{m}-2 i \bar{\omega})} .
\end{aligned}
$$

For the upper time dependent component of the spinor wavefunction, we obtain

$$
\begin{aligned}
& \psi_{u p}(z, t) \simeq e^{-i \omega t}\left[B(1-z)^{\bar{m}} \frac{\Gamma(-2 \bar{m}) \Gamma(1 / 2-2 i \bar{\omega})}{\Gamma(-\bar{m}) \Gamma(1 / 2-\bar{m}-2 i \bar{\omega})}\right. \\
& \left.+B(1-z)^{-\bar{m}} \frac{\Gamma(2 \bar{m}) \Gamma(1 / 2-2 i \bar{\omega})}{\Gamma(\bar{m}) \Gamma(1 / 2+\bar{m}-2 i \bar{\omega})}\right]
\end{aligned}
$$

Now we should impose the boundary condition on the spatial infinity. Taking into account the fact that the background geometry (5) is not asymptotically flat and similarly to the previous case we require that the wavefunction should vanish at infinity. To obey the condition, we should impose the requirement that the argument of the gamma function $\Gamma(1 / 2+\bar{m}-2 i \bar{\omega})$ is equal to nonpositive integer: $1 / 2+\bar{m}-2 i \bar{\omega}=-n$ (the condition which defines the poles of the gamma function). The latter relation allows us to obtain quasinormal frequencies, which take the form

$\omega=-i \frac{u_{+}}{l}\left(n+\bar{m}+\frac{1}{2}\right) ;$

here $n=0,1,2, \ldots$. The quasinormal frequencies are completely imaginary and the imaginary part is negative for any number $n$. Now we conclude that the black hole metric (5) is stable under the influence of the fermionic perturbations. To consider QN modes for the lower component of the wavefunction, we use Eq. (28) which can be rewritten in the form

$$
\begin{aligned}
\psi_{2} & =-\frac{(1-z)}{\bar{m}}\left(\frac{i \bar{\omega}}{\sqrt{z}}+\sqrt{z} \frac{\partial}{\partial z}\right) \psi_{1} \\
& =-\frac{(1-z)}{\bar{m}} z^{1 / 2+i \bar{\omega}} \frac{\partial}{\partial z} z^{i \bar{\omega}} \psi_{1} .
\end{aligned}
$$

As a consequence, the lower component of the wavefunction will be as follows:

$$
\begin{aligned}
\psi_{2}(z)= & -\frac{B \bar{m}}{1 / 2-2 i \bar{\omega}}(1-z)^{\bar{m}} z^{1 / 2-i \bar{\omega}} \\
& \times{ }_{2} F_{1}\left(\bar{m}+\frac{1}{2}-2 i \bar{\omega}, \bar{m}+1, \frac{3}{2}-2 i \bar{\omega} ; z\right) .
\end{aligned}
$$

In the domain near the horizon point (when $z \rightarrow 0$ ), the obtained wavefunction will lead to the ingoing wave as it is required. The behaviour of the function $\psi(z)$ should be investigated at the spatial infinity. To analyze the behaviour at infinity, we make use of the Kummer's transformation again. Making a similar analysis to that performed for the upper component, we conclude that the lower component would have the same quasinormal frequencies (74) as the upper one. We note that the situation that for the lower components we might have the same (as well as additional different) set of frequencies was described in Ref. [36]. Comparing the obtained result for quasinormal frequencies with the corresponding result for the scalar perturbation, we conclude that the fermion field perturbation is stable for arbitrary mass of the fermion field whereas for the scalar field perturbations it might be unstable for sufficiently large masses of the field [34]. It can also be shown that the imposed boundary conditions at infinity lead to the vanishing flux, defined by Eq. (44), so the situation is completely identical to the previous case.

\section{Concluding remarks}

We studied fermionic quasinormal frequencies for two types of $1+1$ dimensional HL black holes. The first type of black hole's solution is similar to the corresponding solution, which can be found in the framework of the standard GR. We impose boundary conditions on the solutions of the Dirac equations to obtain quasinormal modes, namely we demand that in the 
vicinity of the horizon the wavefunction should behave as an ingoing wave and it has to vanish at infinity because the background geometry is not asymptotically flat. The solutions which satisfy the conditions mentioned above were found and the corresponding frequencies of the wavefunctions are complex and the imaginary parts of the frequencies are bounded from above. The obtained continuous spectrum for the quasinormal modes is in agreement with the results of Ref. [39]. As has been mentioned above a continuous QNF spectrum might appear for other types of black hole geometries $[40,41]$. We also note that our analysis leads to the conclusion that the upper and lower components of the Dirac wavefunction have the same spectra.

The second type of black hole solution is defined in the presence of a dilatonic field. For this type of black hole's geometry we impose the same boundary conditions on the solution of the wave equation. The solution which fulfills the imposed boundary condition has a purely imaginary discrete spectrum. It should be noted that the fermion field of arbitrary mass is stable in that geometry and as we mentioned before, for scalar field perturbations it might be unstable for large masses of the field [34].

Acknowledgements This work was partly supported by Project FF30F (No. 0116U001539) from the Ministry of Education and Science of Ukraine and Grant No. 0116 U005055 of the State Fund For Fundamental Research of Ukraine.

Open Access This article is distributed under the terms of the Creative Commons Attribution 4.0 International License (http://creativecomm ons.org/licenses/by/4.0/), which permits unrestricted use, distribution, and reproduction in any medium, provided you give appropriate credit to the original author(s) and the source, provide a link to the Creative Commons license, and indicate if changes were made. Funded by SCOAP ${ }^{3}$.

\section{References}

1. T. Regge, J.A. Wheeler, Phys. Rev. 108, 1063 (1957)

2. F.J. Zerilli, Phys. Rev. D 2, 2141 (1970)

3. F.J. Zerilli, Phys. Rev. Lett. 24, 737 (1970)

4. H.P. Nollert, Class. Quantum Gravity 16, R159 (1999)
5. E. Berti, V. Cardoso, A.O. Starinets, Class. Quantum Gravity 26, 163001 (2009)

6. R.A. Konoplya, A. Zhidenko, Rev. Mod. Phys. 83, 793 (2011)

7. O. Aharony, S.S. Gubser, J. Maldacena, H. Ooguri, Y. Oz, Phys. Rep. 323, 183 (2000)

8. G.T. Horowitz, V.E. Hubeny, Phys. Rev. D 62, 024027 (2000)

9. D.T. Son, A.O. Starinets, JHEP 09, 042 (2002)

10. A.O. Starinets, Phys. Rev. D 66, 124013 (2002)

11. A. Nunez, A.O. Starinets, Phys. Rev. D 67, 124013 (2003)

12. P.K. Kovtun, A.O. Starinets, Phys. Rev. D 72, 086009 (2005)

13. S. Hod, Phys. Rev. Lett. 81, 4293 (1998)

14. O. Dreyer, Phys. Rev. Lett. 90, 081301 (2003)

15. M. Maggiore, Phys. Rev. Lett. 100, 141301 (2008)

16. R.A. Konoplya, Phys. Rev. D 70, 047503 (2004)

17. C. Kiefer, Class. Quantum Gravity 21, L123 (2004)

18. P. Hořava, Phys. Rev. D 79, 084008 (2009)

19. P. Hořava, JHEP 0903, 020 (2009)

20. P. Horava, Phys. Rev. Lett. 102, 161301 (2009)

21. A. Kehagias, K. Sfetsos, Phys. Lett. B 678, 123 (2009)

22. M.-I. Park, JHEP 09, 123 (2009)

23. R.G. Cai, L.M. Cao, N. Ohta, Phys. Rev. D 80, 024003 (2009)

24. E. Kiritsis, G. Kofinas, JHEP 1001, 122 (2010)

25. Y.S. Myung, Phys. Lett. B 678, 127 (2009)

26. R.G. Cai, L.M. Cao, N. Ohta, Phys. Lett. B 679, 504 (2009)

27. Y.S. Myung, Y.W. Kim, Eur. Phys. J. C 68, 265 (2010)

28. G. Koutsoumbas, P. Pasipoularides, Phys. Rev. D. 82, 044046 (2010)

29. S. Chen, J. Jing, Phys. Lett. B 687, 124 (2010)

30. R.A. Konoplya, Phys. Lett. B 679, 499 (2009)

31. E. Varghese, V.C. Kuriakose, Mod. Phys. Lett. A 26, 1645 (2011)

32. E. Varghese, V.C. Kuriakose, Gen. Relativ. Gravity 43, 2757 (2011)

33. R. Becar, P.A. Gonzalez, Y. Vasquez, Int. J. Mod. Phys. D 22, 1350007 (2013)

34. M. Cruz, M. Gonzalez-Espinoza, J. Saavedra, D. Vargas-Arancibia, Eur. Phys. J. C 76, 75 (2016)

35. C. Barcelò, S. Liberati, M. Visser, Liv. Rev. Relativ. 14, 3 (2011)

36. R. Becar, P.A. Gonzalez, Y. Vasquez, Eur. Phys. J. C 74, 2940 (2014)

37. D. Bazeia, F.A. Brito, F.G. Costa, Phys. Rev. D 91, 044026 (2015)

38. R.B. Mann, A. Sheikh, L. Tarasov, Nucl. Phys. B 341, 134 (1990)

39. S. Estrada-Jimenez, A. Lopez-Ortega, A. Lopez-Ortega, Gen. Relativ. Gravity 45, 2239 (2013)

40. J. Natàrio, R. Schiappa, Adv. Theor. Math. Phys. 8, 1001 (2004)

41. C. Barcelo, A. Cano, L.J. Garay, G. Jannes, Phys. Rev. D 75, 084024 (2007)

42. M. Abramowitz, A. Stegun, Handbook of Mathematical Functions (Dover, New York, 1964) 